\title{
THE GENERAL RELATIVISTIC POTENTIAL OF ASTROMETRIC STUDIES AT MICROARCSECOND LEVEL
}

\author{
V. I. ZHDANOV \\ Astronomical Observatory of Kiev University \\ Observatorna St., 3 Kiev 254053 Ukraine
}

\begin{abstract}
The relativistic effects in positioning of distant objects at microarcsecond level are studied. The main points are: statistics of random variations of the image position of a distant radiation source due to the gravitational field of moving stars, motion of the image due to individual invisible gravitators, and possibilities to obtain information on their masses and velocities. The gravitators shifting the object image may be stars of our Galaxy or of a lensing galaxy.
\end{abstract}

\section{Preliminary remarks}

\subsection{INTRODUCTION}

The gravitational lensing theory contains a number of results concerning the impact of relativistic gravity upon electromagnetic wave propagation, which may be useful to obtain independent astrophysical and cosmological information (Schneider et al. 1992; Refsdal \& Surdej 1993). The advances in astrometric techniques for precise positioning of a radiation source image open new possibilities in this direction (Hosokawa et al. 1993; Høg et al. 1994). Following these authors we discuss in this paper the variations of the image position (IP) due to the gravitational field of stars. For typical positions of the source we consider the statistical action of a large number of stars on IP; then we proceed to the cases of the IP location near individual gravitators, that are less probable, but that may produce a more sensible relativistic effect. The gravitators are supposed to be unobservable. This may be of interest when the source and the deflecting star can be observed in different wave bands (radio and optical), and when the deflector is a dark object or a star of another galaxy.

The order of magnitude of the effects discussed would be the same (for comparable values of the impact parameter of the ray from the source with respect to gravitators) in either the case (a) of a distant source image microlensed by stars of a foreground lensing galaxy, or (b) the case of our Galaxy stars.

We deal with the image displacements at the microarcsecond level; these effects are at present almost unattainable, however their consideration demonstrates the needs for the precision astrometry.

\subsection{BASIC RELATIONS AND NOTATIONS}

In case (a) our starting point is the relation for the two-dimensional vector of the IP angular shift due to the gravitational field of a deflector (see, e.g., Schneider et al. 1992)

$$
\vec{\psi}=-4 k_{d s} m r_{m} / r_{m}^{2}
$$


$k_{d s}=D_{d s} / D_{s}$. Here $D_{d s}, D_{d}, D_{s}$ are the angular distances from the deflector to the source, from the observer to the deflector, and from the observer to the source, respectively; $m=G M / c^{2}, M$ is the deflector mass with the position $\mathbf{r}_{p}$ with respect to the unperturbed ray trajectory, $\mathbf{r}_{p}$ being orthogonal to this trajectory and directed towards the deflector, $\mathbf{r}_{m}=r_{m} \mathbf{r}_{p} / r_{p}, \quad r_{p}=\left|\mathbf{r}_{p}\right|$ ， $r_{m}$ is related to $r_{p}$ by the equation:

$$
r_{m}^{2}-r_{p} r_{m}-R_{E}^{2}=0
$$

$R_{E}=\left(4 m k_{d s} D_{d}\right)^{1 / 2}$ being the Einstein radius of the deflector. Equation (2) has two roots that describe two source images. In case of linear microlensing (that is $r_{p} \gg R_{E}$ ) one of these images is faint and, for the main image, one has $\mathbf{r}_{m} \approx \mathbf{r}_{p}$. Formula (1) refers to the case when $r_{m} \ll D_{d}$ and $r_{m} \ll<D_{d s}$, and this is not the case (b) when $k_{d s} \approx 1$ and the contribution of stars far from the ray path may be essential. In the latter case the following relation must be used:

$$
\vec{\psi}=-2\left(m \mathbf{r}_{m} / r_{m}^{2}\right)\left(1+z \cdot\left(z^{2}+r_{p}^{2}\right)^{-1 / 2}\right), \quad z<<D_{S}
$$

for the mass at the point $\left(\mathbf{r}_{p}, z\right)$. We choose $z$-axis in the direction $\mathbf{N}$, where $\mathbf{N}$ is the unit vector pointing to the position of the source (unperturbed IP), the observer being at the origin. Formula (3) is valid also when $r_{p}$ may be of the same order of magnitude as $z=D_{d}<<D_{s}$. Motion of gravitators results in nonzero angular velocity and acceleration that can be recovered from (1) and (3).

\section{Statistical dragging of distant source image by gravitational field of moving stars}

\subsection{PROBABILITY DISTRIBUTION FOR ANGULAR VELOCITIES OF THE IMAGE}

Here we partially use the results of Zhdanov \& Zhdanova (1994) dealing with the angular variations of a distant source IP induced by random gravitators under some restrictions on the gravitator distribution. The relations below generalize these results to rather a general case incorporating both situations (a) and (b). The gravitator distribution is described by the volume density of $\operatorname{stars} f_{V}(\mathbf{r}, z, \mathbf{v}, W, M)$ with the transverse velocity $\mathbf{v}$, longitudinal velocity $W$ and the mass $M$ at the point $(\mathbf{r}, z)$. In the case (a) the dependence upon $W$ and $z$ is not essential.

Under the supposition of linear microlensing the total IP shift is obtained by summing up the contributions of all gravitators. Then, in view of the gravitators' 
motion, differentiating (1) and (3) over the time we obtain the relation for the average velocity of the image

$$
\begin{aligned}
\mathbf{u}_{t}=-2 k \int d M \int d w \int d^{2} \mathbf{v} \int d z \int d^{2} \mathbf{r} m f_{V}(\mathbf{r}, z, \mathbf{v}, w, M) . \\
\quad\left\{r ^ { - 2 } [ \mathbf { v } - 2 \mathbf { r } ( \mathbf { r } \cdot \mathbf { v } ) / r ^ { 2 } ] \cdot \left[1+z\left(z^{2}+r^{2}\right)^{-1 / 2}+\right.\right. \\
\left.\left.\quad+\left(z r^{2} / 2\right) \cdot\left(z^{2}+r^{2}\right)^{-3 / 2}\right]+(w \mathbf{r}-z \mathbf{v} / 2) \cdot\left(z^{2}+r^{2}\right)^{-3 / 2}\right\},
\end{aligned}
$$

which can be simplified in the case (a) ( $k=k_{d s}$ ) by assuming $r<<z$ and recalculating the result in terms of the surface density of stars in the gravitator plane $z=D_{d}$. In the case (b) we assume $k=1$; here the dependencies upon longitudinal velocity components and upon $Z$ are significant. The singular points in the integral do not lead to any troubles.

We obtained the probability distribution of angular image velocities describing the action of a large number of gravitators. The Markov method has been used to obtain the characteristic function of this distribution. For a typical range of its independent variable, $y$, it is approximated by the expression

$$
\exp \left[-u_{0} y+i\left(\mathbf{u}_{t} \mathbf{y}\right)\right] \text {, where } y=|\mathbf{y}|, \quad u_{0}=4 \pi k \int d M \int d^{2} \mathbf{v} m \cdot|\mathbf{v}| \cdot f_{s}(\mathbf{v}, M) ，
$$

$f_{S}(\mathbf{v}, M)=\int_{0}^{D_{s}} d z \int_{-\infty}^{\infty} d w f_{V}(0, z, \mathbf{v}, w, M)$.

Therefore, for a realistic $f_{V}$ we obtain the velocity distribution valid for both cases (a) and (b):

$$
\mathbf{P}(\mathbf{u})=\frac{u_{0}}{2 \pi}\left[u_{0}^{2}+\left(\mathbf{u}-\mathbf{u}_{t}\right)^{2}\right]^{-3 / 2}
$$

As it can be seen from the relations, in the case of a nonuniform gravitator distribution and a nonzero bulk velocity, there is an average dragging of the image with the velocity $\mathbf{u}_{t}$, depending upon the input of stars far from the ray. The other parameter $u_{0}$ is determined by the masses in the close vicinity of the ray path; its order of magnitude for a gravitator volume density $0.1 \mathrm{pc}^{-3}$ along the ray path of $10 \mathrm{kpc}$ may be estimated as:

$u_{0} \approx 1^{\prime \prime} \cdot 10^{-7}(10 \mathrm{yr})^{-1} k M_{c h} v_{c h} /\left(M_{\odot} \cdot 100 \mathrm{~km} / \mathrm{s}\right)$,

that involves the characteristic values of the gravitator mass and velocity. It is rather small, but distribution (4) decreases slowly, and there is, e.g., $1 \%$ of events with $u \geq 100 u_{0}$. The parameter $\mathbf{u}_{t}$ is roughly of the same order of magnitude, but it depends on the form of the star density distribution. Then, as it can be seen from (4), the presence of bulk gravitator motions amplifies the probability of dragging with the characteristic values of $u$, but it is not important for distribution at large $u$.

For (b), the Galaxy rotation produces an anisotropy in the velocity distribution of the remote radiation sources close to the Galaxy plane. The dragging due to 
the Galaxy stars seems to play a negative role. Indeed, the above characteristic values appear to be comparable to the estimates of proper angular velocities of quasars and, provided that the corresponding accuracy can be achieved, it would be difficult to separate them in observations. However, for (a), the effect might be interesting in precise positioning of different images of the same quasar, when the characteristic parameters may be increased by one or two orders of magnitude because of a larger bulk velocity of the lensing galaxy motion and a more dense region near the ray path. This is the case of some gravitational lenses, e.g., the "Einstein Cross" $2237+0305$. On the other hand, the proper motion of the source may be ruled out in relative angular velocity measurements for different source images.

\subsection{PROBABILITY DISTRIBUTION FOR ACCELERATIONS}

The motion of foreground stars causes the angular acceleration $\mathbf{a}$ of the distant source image. To obtain the distribution $\mathbf{P}_{A}(\mathbf{a})$, one may neglect the accelerations of the stars. As distinct from (4), the acceleration distribution turns out to be determined only by the stars near the ray path and relation (1) may be used from the very beginning. The characteristic function of the two-dimensional variable $y$

is $\exp \left[-\left(a_{0}|\mathbf{y}|\right)^{2 / 3}\right]$, where

$$
a_{0}=k\left\{6 \sqrt{\pi} \Gamma(5 / 6) \int d M \int d^{2} \mathbf{v} m^{2 / 3}|\mathbf{v}|^{4 / 3} f_{s}(\mathbf{v}, M)\right\}^{3 / 2} .
$$

This yields the distribution written here in the form suitable for the estimates for large values of $a=|\mathbf{a}|$ :

$\mathbf{P}_{a}(\mathbf{a})=\frac{1}{3 \pi} \sum_{n=1}^{\infty} \sin (\pi n / 3) \cdot[n \Gamma(n / 3)]^{2} \frac{(-1)^{n+1}\left(2 a_{0}\right)^{2 n / 3}}{n ! a^{2 n / 3+2}}$

The numerical estimate, for the same values of the volume density and the ray path as in sect. 2.1, is given by the formula

$a_{0} \approx 1^{\prime \prime} \cdot 10^{-8}(10 y r)^{-2}\left\{k M_{c h} v_{c h}^{2} /\left(M_{\odot} \cdot(100 k m / s)^{2}\right)\right\}$.

As before, this value could be more significant in the case (a). From (5) we infer that there is about $3 \%$ of events with $a \geq 100 a_{0}$. Note that in case (b) of a differential measurement with two distant sources, these must be separated by an angular distance exceeding the characteristic one between the Galaxy stars to avoid correlations. If the IP variation is measured with respect to some reference Galaxy star close to IP, then the integrations over $Z$ in the above expressions must be performed from the positions of this star.

\section{Gravitational image motion due to an individual deflector, and its mass, distance and velocity}

The observations of the relative motion of the source image with respect to the deflecting star due to the gravitational parallactic effect would allow one to 
measure the star mass and distance (Hosokawa et.al 1993). These authors pointed out that this effect must be studied jointly with the image motion owing to the motion of a gravitator considered by Chollet (1979) and Kovalevsky et al. (1986). Here we present a simple argument that observation of both effects could yield the values of the mass and the velocity of the invisible gravitator (linear microlensing, $\mathbf{r}_{m} \approx \mathbf{r}_{p}$ ). A more detailed consideration of the question involves evaluations by the nonlinear least square method. We restrict our consideration to show that the above parameters may be determined in principle. The way used below is not the best one for the real data treatment, but it makes evident the formal solvability of the problem.

Here the source is assumed to be at rest. The observer's shift by the vector $\mathbf{p}$ in a transverse plane orthogonal to $\mathbf{N}$ gives rise to the corresponding change in the relative position of the gravitator with respect to the unperturbed ray trajectory $\mathbf{r}_{p} \rightarrow \mathbf{r}_{p}+k_{d s}^{*} \mathbf{p}$, the coefficient $k_{d s}^{*}$ depending upon background space-time (in flat space-time $k_{d s}^{*}=k_{d s}$ ). For the gravitator velocity, $\mathbf{v}_{g}$, in the plane orthogonal to $\mathbf{N}$, one can write $\mathbf{r}_{p}=\mathbf{r}_{\text {ort }}+\left(\mathbf{v}_{g}+k_{d s}^{*} \mathbf{v}_{S}\right)\left(t-t_{m}\right)+k_{d s}^{*} \mathbf{p}_{\text {orb }}$, where we have separated the linear motion due to the velocity of the Sun, $\mathbf{v}_{S}$, and the periodic orbital motion of the observer; the vector $r_{\text {ort }}$ is orthogonal to $\mathbf{v}_{g}+k_{d s}^{*} \mathbf{v}_{S}$ and corresponds to the minimum distance from the ray to the gravitator at an epoch $t_{m}$ in absence of the orbital motion. Under the above condition of the linear microlensing, the straight line motions of the gravitator and of the observer induce the apparent motion of the image exactly along a circle. The actual source position is represented by the limiting point, $\mathbf{S}_{0}$, on the circle that corresponds to an infinite position of the gravitator. The gravitational shift of the IP from the unperturbed position $S_{0}$ is given by the angle $\vec{\psi}$ from (1), and the epoch $t_{m}$ is determined at the maximum value of this angle $\psi_{\max }=4 \mathrm{~m} / \mathrm{r}_{\mathrm{ort}}$.

Using (1) we have

$$
\vec{\psi} /|\vec{\psi}|^{2}=\left[\mathbf{r}_{\text {ort }}+\left(\mathbf{v}_{g}+k_{d s}^{*} \mathbf{v}_{S}\right)\left(t-t_{m}\right)+k_{d s}^{*} \mathbf{p}_{\text {orb }}\right] /\left(4 m k_{d s}\right) \text {. }
$$

Separating the linear terms from the periodic ones (with known porb) one obtains the combination $k_{d s}^{*} /\left(k_{d s} M\right)$ and, for $k_{d s}^{*}=k_{d s}$, the inverse mass. For an infinite source we have $k_{d s}^{*}=k_{d s}=1$ and from the above equation we find $\mathbf{v}_{g}+k_{d s}^{*} \mathbf{v}_{\mathrm{S}}$ and $\mathbf{r}_{\text {ort }}$ which defines the IP circle diameter. For known $k_{d s}^{*} \mathbf{v}_{\mathrm{S}}$ this allows us to find the transversal velocity of the gravitator. In the example by Hosokawa et al. the condition of linear microlensing is satisfied, $\psi_{\text {max }} \approx 0.7$ milliarcsec, and the gravitational IP shift due to the observer's annual motion is estimated to be about 10 microarcsec. 
If $r_{p}$ and $R_{E}$ are of the same order of magnitude, the following points should be mentioned: (i) due to microlensing there is a high amplification of the source images, which yields an additional source of information; (ii) the Einstein radius $R_{E}$ appears in photometric and astrometric effects. This leads to the results of Høg et al. (1994), who have shown that combination of photometric and astrometric measurements during microlensing of a star in the Large Magellanic Cloud (LMC) by a massive compact halo object is sufficient to determine the mass, proper motion and distance of this gravitator, provided that the position, distance and relative motion of the source with respect to the observer are known. The probability of this event is very small, but the examination of millions of stars in the LMC, proposed by Paczinsky (1986), makes these observations possible, and such events have already been detected (Alcock, 1993; Aubourg, 1993). The order of magnitude of $\psi_{\max }$ during the event is milliarcsecond.

The brightness of two source images during microlensing is comparable to each other and the interpretation of observations depends on whether it is possible to resolve them. In the case of a positive answer, there is one more opportunity to obtain information: (iii) one may hope to estimate the angular separation

of the images $\Delta \psi=\left(r_{p}^{2}+R_{E}^{2}\right)^{1 / 2} / D_{d}$, which (in this case) is roughly of the same order of magnitude as $\psi_{\max }$.

There is a considerable probability of microlensing events in gravitational lenses (Schneider et al. 1992; Refsdal \& Surdej, 1994). The angular separation of the images due to the gravitational field of an isolated star and the corresponding $\psi_{\max }$ are of the order of

$R_{E} / D_{d} \approx 1^{\prime \prime} \cdot 10^{-5} \cdot\left[k_{d s} \cdot M \cdot 10^{8} \mathrm{pc} /\left(M_{\odot} D_{d}\right)\right]^{1 / 2}$.

This value may be enhanced by gravitational field of the lensing galaxy.

\section{References}

Alcock, C., Akerloff, C.W., Allsman, R.A., et al., 1993, Nature 365, 621

Aubourg, E., Bareyre, P., Brehin, S., et al., 1993, Nature 365, 623

Chollet, F., 1979, C.R. Acad. Sci. Paris, Ser.B 288, 163

Høg, E., Novikov, I.D., Polnarev, A.G., 1994, MACHO photometry and astrometry. NORDITA prepr. 94/26 A

Hosokawa, M., Ohnishi, K., Fukushima, T., Takeuti, M., 1993, A\&A 278, L27

Katz, N., Balbus, S., Paczinsky, B., 1986, ApJ 306, 2

Kovalevsky, J., Mignard, F., Froschle, M. 1979. Proc. IAU Symp.114,

Eds.: J.Kovalevsky and V.A.Brumberg, p.369.

Paczinsky, B. 1986, ApJ 304, 1

Refsdal, S., Surdej J., 1994, Repts. Progr. Phys. 56, 117.

Schneider, P., Ehlers, J., Falco, E.E., 1992, Gravitational Lensing.

Springer, New York

Zhdanov, V.I., Zhdanova, V.V., 1994, Analytical relations for time-dependent statistical microlensing. Submitted to A\&A. 